\title{
Bending-induced extension in two-dimensional crystals
}

\author{
Douxing Pan ${ }^{1,2}$ - Yao $\mathrm{Li}^{3}$ - Tzu-Chiang Wang ${ }^{1} \cdot$ Wanlin $\mathrm{Guo}^{3}$
}

Received: 7 June 2016 / Revised: 7 July 2016 / Accepted: 11 July 2016 / Published online: 10 November 2016

(C) The Chinese Society of Theoretical and Applied Mechanics; Institute of Mechanics, Chinese Academy of Sciences and Springer-Verlag Berlin Heidelberg 2016

\begin{abstract}
We find by ab initio simulations that significant overall tensile strain can be induced by pure bending in a wide range of two-dimensional crystals perpendicular to the bending moment, just like an accordion being bent to open. This bending-induced tensile strain increases in a power law with bent curvature and can be over $20 \%$ in monolayered black phosphorus and transition metal dichalcogenides at a moderate curvature of $2 \mathrm{~nm}^{-1}$ but more than an order weaker in graphene and hexagon boron nitride. This accordion effect is found to be a quantum mechanical effect raised by the asymmetric response of chemical bonds and electron density to the bending curvature.
\end{abstract}

Keywords Monolayer black phosphorus - Bending to extension - Accordion effect - Density functional theory

Douxing Pan and Yao Li have contributed equally to this work.

Electronic supplementary material The online version of this article (doi:10.1007/s10409-016-0602-2) contains supplementary material, which is available to authorized users.

Tzu-Chiang Wang

tcwang@imech.ac.cn

$\triangle$ Wanlin Guo

wlguo@nuaa.edu.cn

1 State Key Laboratory of Nonlinear Mechanics, Institute of Mechanics, Chinese Academy of Sciences, Beijing 100190, China

2 University of Chinese Academy of Sciences, Beijing 100049, China

3 State Key Laboratory of Mechanics and Control for Mechanical Structures and Key Laboratory for Intelligent Nano Materials and Devices (MOE), Nanjing University of Aeronautics and Astronautics, Nanjing 210016, China

\section{Introduction}

Graphene and graphene-like two-dimensional (2D) crystals have rapidly emerged in the past decade as the most promising materials with exceptional physical behaviors $[1,2]$. The most unique characters of the $2 \mathrm{D}$ crystals are their atomic thinness and extremely high flexibility [2,3]. It is widely known that the electronic and optical performances of such 2D crystals are extremely sensitive to flexion deformation [4-9]. For example, graphene is regarded as the strongest material in the world with a Young's modulus of 1 terapascal [10], and the Young's modulus of monolayered $\mathrm{MoS}_{2}$ is comparable to that of steel [11], but they are extremely easy to bend and ripple and can even be directly rolled into nanotubes [12]. Most recently, an interesting bending Poisson effect was revealed in a series of $2 \mathrm{D}$ crystals where the bending deformation is found to be able to induce deformations along the bending moment [13]. The 2D monolayers are not only flexible, they are also very stable under serious bending deformation, or they can be seriously folded without breaking. The graphene and hexagonal boron nitride (h-BN) monolayers can be stable to a curvature radius down to around $0.15 \mathrm{~nm}$ $[14,15]$ and the black phosphorus (BP), or orthorhombic black phosphorus (OBP) monolayer can be stable to a curvature radius of $0.5 \mathrm{~nm}$ when bending along a zigzag and much smaller along an armchair direction [16]. However, detailed bending behaviors, especially coupling between flexion and in-plane deformation in 2D crystals, remain poorly understood and have never been reported in many 2D crystals, although some interesting coupling between in-plane stretching and bending or twisting behaviors has been reported in graphene and its ribbon structures [17-23]. In this article, we show by ab initio simulations that bending $2 \mathrm{D}$ crystals can induce significant overall tensile deformations perpen- 
dicular to the bending moment, just like an accordion being bent to open. The underlying mechanism is revealed through comprehensive investigations of the responses of chemical bonds and electron density to bending curvature. Interestingly, such an accordion effect is found to be intrinsic to all the $2 \mathrm{D}$ crystals investigated in this work.

\section{Methods}

In what follows, the results of BP monolayers are used to show how the accordion effect occurs before the intrinsic accordion effect in a wide range of $2 \mathrm{D}$ crystals that are presented. The geometrical relaxations are carried out within the framework of density-functional theory (DFT) [24], as implemented in the Vienna ab initio simulation package (VASP) code $[25,26]$. The generalized gradient approximation in the form of the Perdew-Burke-Ernzerhof exchange correlation functional [27] and projector augmented wave potentials are used [28-30]. High-accuracy settings are adopted in the geometrical relaxations. ${ }^{1}$ The DFT molecular dynamic simulations are carried out with a $1 \times 1 \times 3$ supercell and $1 \times 1 \times 3 k$-point mesh at $300 \mathrm{~K}$ in the NVT ensemble for $2400 \mathrm{fs}$ (the time step length is $2 \mathrm{fs}$ ).

The relaxed lattice constants of the BP unit cell are $a_{1}=$ $0.4620 \mathrm{~nm}, a_{2}=0.3299 \mathrm{~nm}$, and the length $L_{0}$ of a planar strain-free BP monolayer can be written as

$L_{0}=\left(n^{2}\left|\boldsymbol{a}_{1}\right|^{2}+n m\left|\boldsymbol{a}_{1}\right|\left|\boldsymbol{a}_{2}\right|+m^{2}\left|\boldsymbol{a}_{2}\right|^{2}\right)^{\frac{1}{2}}$,

where $n$ and $m$ are the number of BP unit cells along the armchair and zigzag directions, respectively. To mimic the pure bending deformation, BP monolayers are rolled into corresponding closed black phosphorus nanotubes (PNTs) $[14]$ and the reciprocal of the radius is defined as the bending curvature (Fig. 1). When the BP monolayer is rolled into an armchair PNT (aPNT), it is called armchair bending, or armbending; when rolled into a zigzag PNT (zPNT), it is called zigzag bending, or zig-bending. A periodical condition will be applied along the axial direction of the nanotubes in the following DFT simulations.

Based on DFT simulation results, the tensile and compressive strains induced by pure bending in the outer and inner half-layers of BP are defined as

\footnotetext{
1 The energy and force convergence criteria are set at $10^{-4} \mathrm{eV}$ and $0.1 \mathrm{eV} / \mathrm{nm}$, respectively. The kinetic energy cutoff for the plane wave basis set is adopted as $450 \mathrm{eV}$, which guarantees that the absolute energies converge to around $2 \mathrm{MeV}$. The reciprocal space for the unit cell of $\mathrm{BP}$ is meshed at $14 \times 10 \times 1$ using Monkhorst-Pack meshes centered at the $\Gamma$ point. For one-dimensional periodic cells, a vacuum space of $2 \mathrm{~nm}$ is included for all tubes and a dense $k$-point mesh $(1 \times 1 \times 12)$ is used for Brillouin zone sampling. The lattice constants in all directions are fully optimized for all tubes.
}

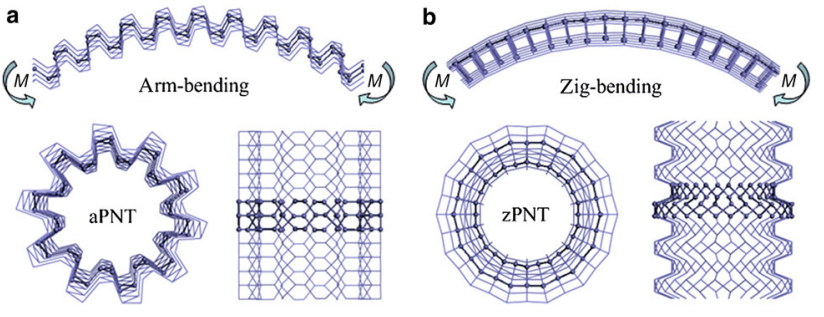

Fig. 1 Monolayered BP in bent state. a Arm-bending, with bending moment $M$ being perpendicular to armchair direction, and an armchair BP nanotube (aPNT). b Zig-bending, with bending moment $M$ being perpendicular to zigzag direction, and a zigzag BP nanotube (zPNT)

$\varepsilon_{\mathrm{t}}=\frac{L_{\mathrm{out}}-L_{0}}{L_{0}}$ and $\varepsilon_{\mathrm{c}}=\frac{L_{\mathrm{in}}-L_{0}}{L_{0}}$,

respectively, where $L_{\text {out }}$ and $L_{\text {in }}$ are the length of the outer and inner half-layers of the BP monolayer in the bent state as defined in Fig. S1 in the supplementary material. ${ }^{2}$ The tensile strain of the midplane of the bent BP monolayer can be defined as

$\varepsilon_{\mathrm{m}}=\frac{\varepsilon_{\mathrm{t}}+\varepsilon_{\mathrm{c}}}{2}$,

which presents the overall tensile deformation due to bending. In this work, the curvature means the curvature of the midplane of the bent monolayer.

\section{Results and discussion}

Figure 2 shows that the bent BP monolayer exhibits apparent tensile strain in its outer half-layer and compressive strain in its inner half-layer perpendicular to the bending moment or the axial direction of the PNT. However, the tensile strain within the outer half-layer is significantly larger than the compressive strain within the inner half-layer (Fig. 2a,b), resulting in a significant overall tensile deformation perpendicular to the bending moment (Fig. 2c). This bending-induced overall extension is impossible in the classical theory of elasticity [31], where a plate subjected to a pure bending moment always deflects symmetrically with tensile strain in its outer side and equal compressive strain in its inner side. Such an asymmetric deformation is similar to an accordion being bent to open.

A comparison of Fig. $2 \mathrm{a}$ and $\mathrm{b}$ shows that zig-bending induces a larger extension and smaller compression than arm-

\footnotetext{
${ }^{2}$ See supplemental material for the determination of the lengths of the outer and inner half-layers of bent BP monolayers, bending-induced asymmetrical partial charge distribution, the bending to tensile deformation in other transitional metal dichalcogenides, the influence of finite temperature on the bending deformation, and the bending rigidity of monolayered BP with fixed midplane length.
} 

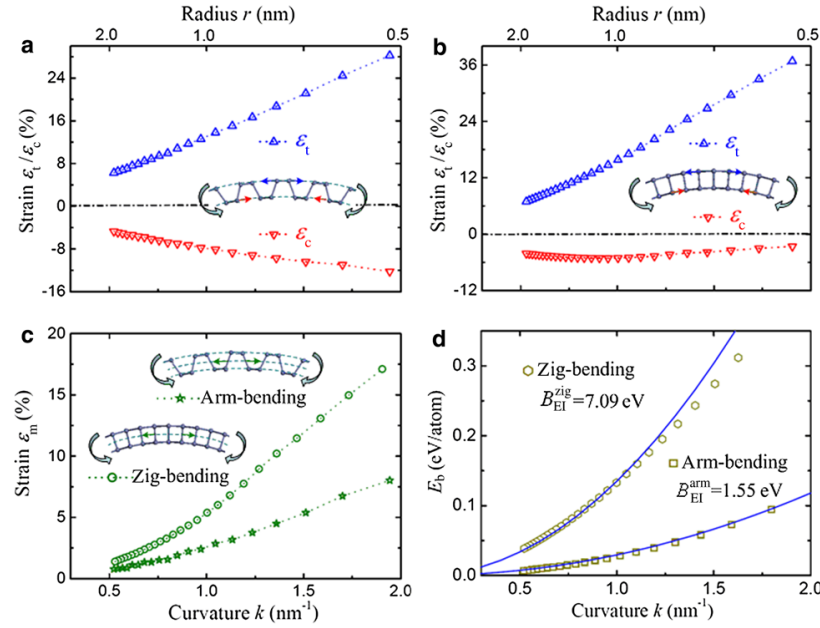

Fig. 2 Bending-induced extension in monolayered BP. a Armbending- and $\mathbf{b}$ zig-bending-induced tensile and compressive strains in outer and inner half-layers as a function of curvature. $\mathbf{c}$ Tensile strain of midplane in bent state. d Bending strain energy, from which bending moduli can be obtained. The dashed and solid curves in plots $\mathbf{c}$ and $\mathbf{d}$ are the power-law and quadratic fitting of the DFT results for $k<1.5 \mathrm{~nm}^{-1}$, respectively

bending. For zig-bending, the change of tensile and compressive strain with the bending curvature $k$ exhibits more nonlinearity. The compressive strain even decreases with further increases in the curvature beyond $1 \mathrm{~nm}^{-1}$. This shows that the accordion effect in the BP monolayer is anisotropic when the midplane strain under zig-bending is 2.27 times that under arm-bending at a fixed bending curvature of $1.36 \mathrm{~nm}^{-1}$.

When the bending curvature is as low as around $0.5 \mathrm{~nm}^{-1}$, the tensile and compressive strains are $\varepsilon_{\mathrm{t}}=6.26 \%$ and $\varepsilon_{\mathrm{c}}=$ $-4.71 \%$ for arm-bending, and $\varepsilon_{\mathrm{t}}=6.93 \%$ and $\varepsilon_{\mathrm{c}}=-4.14 \%$ for zig-bending, respectively. At the bending curvature near $2 \mathrm{~nm}^{-1}$, the tensile and compressive strains for arm-bending are $\varepsilon_{\mathrm{t}} \approx 28.25 \%$ and $\varepsilon_{\mathrm{c}}=-12.22 \%$, and for zig-bending $\varepsilon_{\mathrm{t}} \approx 36.82 \%$ and $\varepsilon_{\mathrm{c}}=-2.62 \%$, respectively. The extensile strain $\varepsilon_{\mathrm{m}}$ reaches $8.00 \%$ and $17.04 \%$ under arm- and zigbending to curvature of $2 \mathrm{~nm}^{-1}$, respectively, which cannot be simply explained by an asymmetrical in-plane uniaxial tensile and compressive deformation of the BP monolayer (supplemental material, Figs. S2 and S3 (footnote 2)).

Figure $3 \mathrm{a}-\mathrm{c}$ shows the variation in bond lengths and angles in the BP monolayer with curvature under arm-bending. It can be seen from Fig. $3 b$ that as the curvature increases, the bond angle $\alpha_{\text {out }}$ increases more significantly than the decrease in $\alpha_{\text {in }}$. Thus, it is more difficult to compress the inner half-layer than to stretch the outer half-layer. Figure $3 \mathrm{c}$ shows that the bond lengths $d_{\text {out }}$ and $d_{\mathrm{m}}$ increase while $d_{\text {in }}$ decreases with increasing curvature. As a result, the faster increasing $\alpha_{\text {out }}$ and increase in both $d_{\mathrm{m}}$ and $d_{\text {out }}$ will dramatically extend the length of the outer half-layer $L_{\text {out }}$ and the length of the midplane, although the decrement in $\alpha_{\text {in }}$ and $d_{\text {in }}$ will slightly shorten the length of the inner half-layer $L_{\text {in }}$.
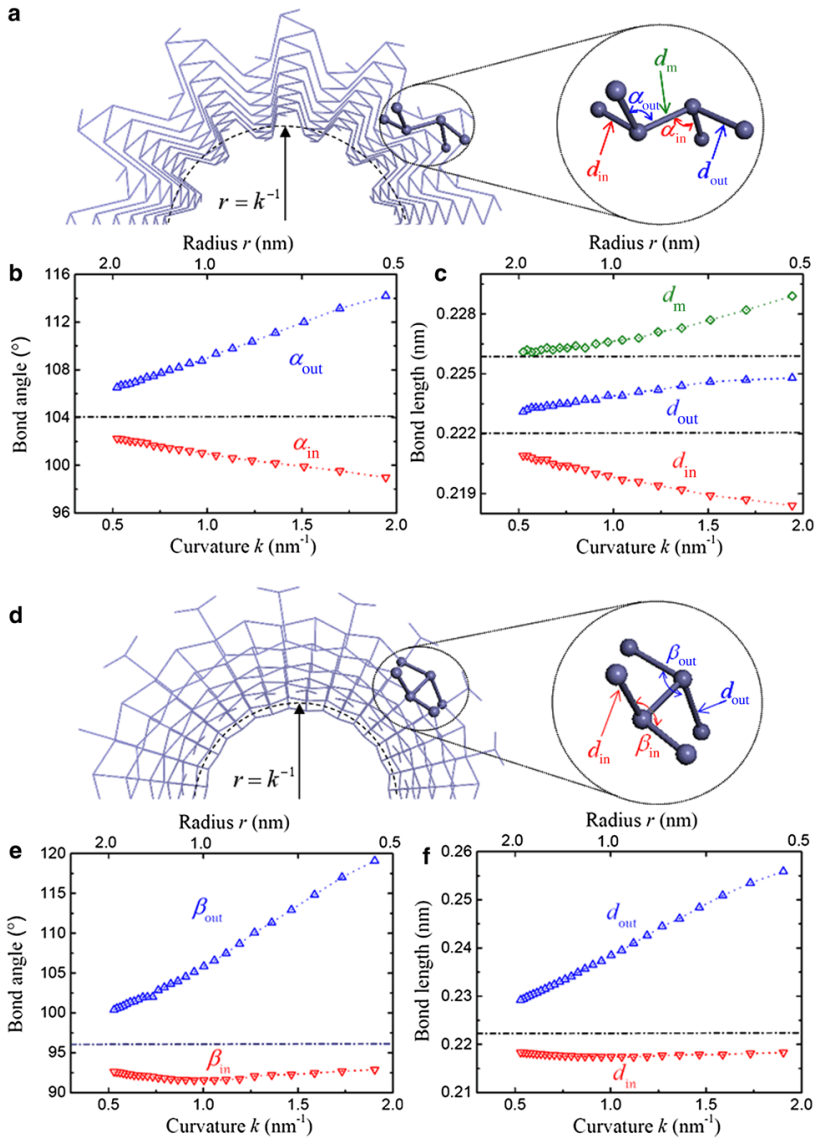

Fig. 3 Bonding structures in monolayered BP under pure bending. a-c Arm-bending. a Bonding structures. b Bond lengths and $\mathbf{c}$ bond angles change with bending curvature. The corresponding results for zig-bending are presented in plots $\mathbf{d}-\mathbf{f}$. The dot-dash lines in plots b-f denote the corresponding values in the planar strain-free BP monolayer

Similarly, for the BP monolayer under zig-bending, as shown in Fig. $3 \mathrm{~d}-\mathrm{f}$, the bond angle $\beta_{\text {out }}$ notably increases with increasing curvature, while $\beta_{\text {in }}$ decreases slightly and even starts to decrease with further increases in the curvature beyond approximately $1 \mathrm{~nm}^{-1}$. Similar trends can be observed for bond lengths $d_{\text {out }}$ and $d_{\text {in }}$ in Fig. 3f. The nonmonotonic changes in the bond angle $\beta_{\text {in }}$ and bond length $d_{\text {in }}$ lead to a more significant accordion effect in zig-bending BP. As will be shown in what follows, this asymmetric variation in the bonding structure in the bent BP monolayer can be further attributed to a bending-induced shift in electron density.

In a planar BP monolayer, the projected density of electrons (PDOE) is symmetric about the midplane of the monolayer, as shown by Fig. $4 \mathrm{a}$ and $\mathrm{b}$ from both the armchair and zigzag directions (supplemental material, Fig. S4 (footnote 2)). Under arm- and zig-bending, the PDOE becomes asymmetric about the midplane, as shown by Fig. $4 \mathrm{c}$ and d, respectively. The higher electron density in the compressive side will constrain the compressive strain, as the high-density 

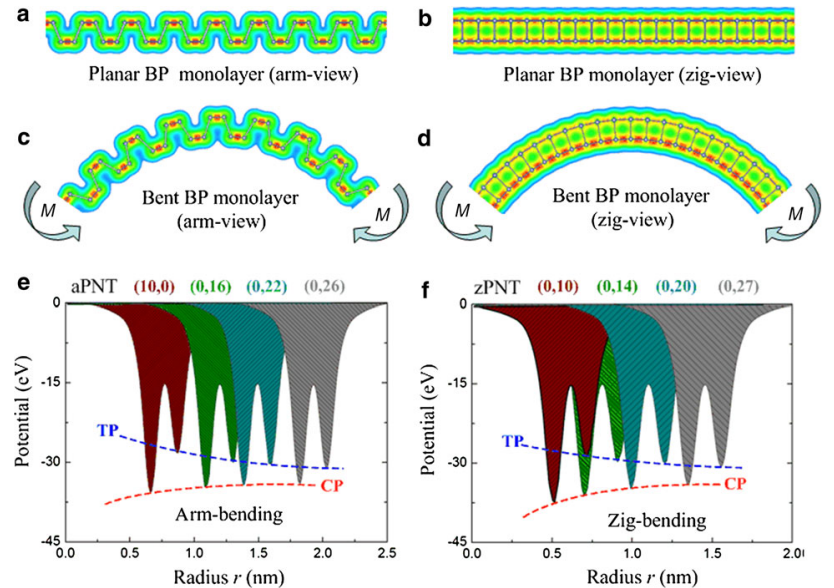

Fig. 4 Projected density of electron (PDOE) and distribution of chemical potential. a, b PDOE for planar BP monolayer viewed from a armchair direction and $\mathbf{b}$ zigzag direction. $\mathbf{c}$ PDOE for BP monolayer under arm-bending and d zig-bending at same bending curvature. Saturation value of PDOE is $0.1 \mathrm{e} / \mathrm{Bohr}^{3}$ in plots a, c, and $0.065 \mathrm{e} / \mathrm{Bohr}^{3}$ in plots b, d. e, f Deformation potential along thickness of BP monolayer under bending. e Arm-bending and $\mathbf{f}$ zig-bending. The variation curves of the lowest potential on the tensile side (TP) and that on the compressive side (CP) with radius are drawn in plots $\mathbf{e}, \mathbf{f}$

electrons tend to repel each other. Figure $4 \mathrm{e}$ and $\mathrm{f}$ shows increasingly asymmetric chemical potentials with a decreasing curvature radius under arm- and zig-bending. The chemical potential on the tensile side (TP) increases with increasing curvature, while the chemical potential on the compressive side (CP) decreases with a decreasing curvature radius. The induced potential differences will result in a built-in electrical field. As a result, the high electron density on the compressive side and the formation of the built-in electrical field will limit the compressive strain and so extend the length of the midplane, just like bending to open an accordion. A comparison of the results in Fig. 4e and $\mathrm{f}$ shows that the induced potential difference is slightly larger under zig-bending than under arm-bending. This larger potential difference can explain the stronger accordion effect under zig-bending (Fig. 2).

Our density-functional calculations also show that the band gap of a bent BP monolayer decreases with increasing bending curvature along both armchair and zigzag directions, with the valence band maximum rising and conduction band minimum decreasing, and the electron densities corresponding to the valence band maximum becoming asymmetric about the midplane in a way similar to that of the PDOE shown in Fig. 4c and d (Fig. S5 in supplemental material (footnote 2)). The energy band structures and partial charge densities corresponding to the valence band maximum of a planar BP monolayer are presented in Fig. S4 in the supplemental material (footnote 2).
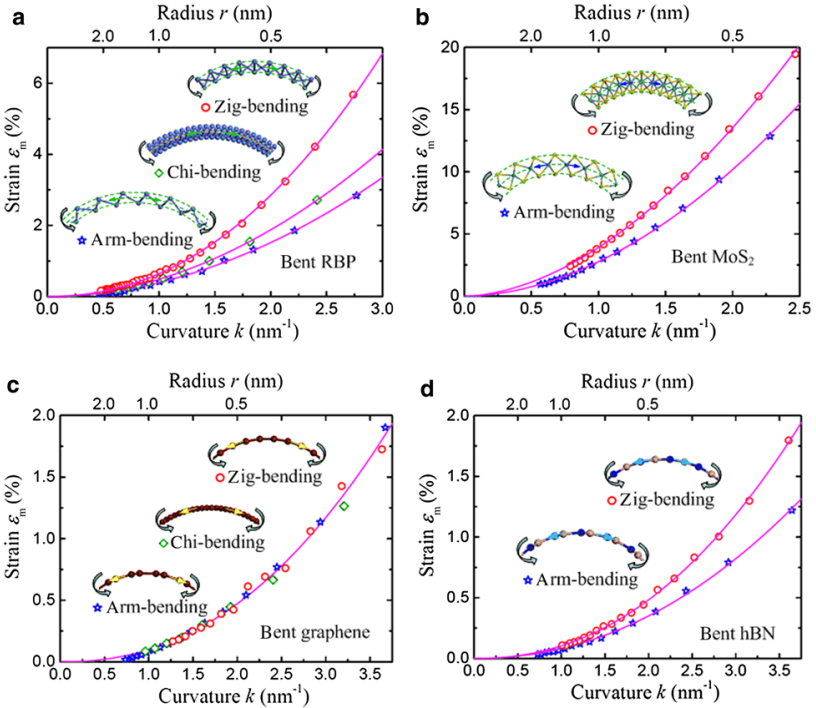

Fig. 5 Bending to tensile deformation in other two-dimensional crystals. a RBP. b $\mathrm{MoS}_{2}$. c Graphene (GR). d h-BN. Here, chi-bending means the monolayers rolled up into $(2 n, n)$ nanotubes. Points DFT results; solid lines power-law fitting curves, with $c$ and $\lambda$ listed in Table 1

Extensive DFT-based similar modeling and calculations show that the revealed accordion effect is intrinsic to monolayered blue phosphorus, or rhombohedral black phosphorus (RBP) and transition metal dichalcogenides (TMDCs), as shown by the results in Fig. 5 and the supplemental material, Fig. S6 (footnote 2). The bending-induced midplane tensile strains in monolayered molybdenum disulfide $\left(\mathrm{MoS}_{2}\right)$ and other transition metal dichalcogenides (namely, $\mathrm{WS}_{2}, \mathrm{MoSe}_{2}$ and $\mathrm{WSe}_{2}$ ) are almost as strong as that in OBP but becomes much weaker in RBP, only around one third of that in OBP. A similar phenomenon to that shown in Fig. 5c was noticed by Zólyomi [17] in his $\mathrm{PhD}$ thesis during the structural optimization of carbon nanotubes using DFT calculations. In monolayered graphene (GR) and h-BN, the bending-induced tensile midplane strains become one order lower, where the flexion-induced asymmetric $\pi$-electron density should be the only reason for the accordion effect. In all cases except graphene, the accordion effect under zig-bending is more significant than under arm-bending. Therefore, the accordion effect is closely dependent on the elements, atomic structure, chirality, and thickness of 2D crystals.

It is interesting that the midplane tensile strain $\varepsilon_{\mathrm{m}}$ always increases as a simple power function of the bending curvature $k$,

$\varepsilon_{\mathrm{m}}=c k^{\lambda}$.

The power-law fitting curves are drawn in Fig. 5 and Fig. S6 in the supplemental material (footnote 2), using the fitting coefficient $c$ and exponent $\lambda$ listed in Table 1. It can be seen that the DFT results for all the 2D crystals can be primely fitted by the 
Table 1 Fitted coefficient $c$ and exponent $\lambda$ in power law $\varepsilon_{\mathrm{m}}=c k^{\lambda}$

\begin{tabular}{|c|c|c|c|c|c|c|c|c|c|}
\hline 2D crystals & & GR & $\mathrm{BN}$ & RBP & OBP & $\mathrm{MoS}_{2}$ & $\mathrm{WS}_{2}$ & $\mathrm{MoSe}_{2}$ & $\mathrm{WSe}_{2}$ \\
\hline \multirow[t]{2}{*}{ Arm-bending } & $c$ & 0.10 & 0.08 & 0.39 & 2.55 & 2.77 & 2.82 & 4.24 & 4.50 \\
\hline & $\lambda$ & 2.24 & 2.10 & 1.95 & 1.85 & 1.88 & 2.14 & 2.04 & 2.09 \\
\hline \multirow[t]{2}{*}{ Chi-bending } & $c$ & 0.10 & & 0.49 & & & & & \\
\hline & $\lambda$ & 2.24 & & 1.95 & & & & & \\
\hline \multirow[t]{2}{*}{ Zig-bending } & $c$ & 0.10 & 0.14 & 0.64 & 5.33 & 3.91 & 4.05 & 5.79 & 5.84 \\
\hline & $\lambda$ & 2.24 & 2.21 & 2.15 & 2.07 & 1.79 & 2.12 & 2.10 & 2.06 \\
\hline Thickness $(\AA)^{\mathrm{a}}$ & $t$ & 0.00 & 0.00 & 1.24 & 2.10 & 3.13 & 3.14 & 3.34 & 3.35 \\
\hline
\end{tabular}

a The thickness here is the atomic center distance of the top and bottom atomic layers of the planar $2 \mathrm{D}$ crystals, $1 \AA=0.1 \mathrm{~nm}$

power law with exponents around 2. As a similar power law has been found for the bending Poisson effect in 2D crystals [13], it can be expected that there should be a linear relationship between bending-induced tensile strain and bendinginduced axial strain in most 2D crystals. The slight change in the exponent should reflect a change in the sensitivity of the bending effects to the atomic structures and electronic properties of 2D crystals. Because the BP monolayer can become instable under strong bending deformation [16], the $\varepsilon_{\mathrm{m}} \sim k$ curves deviate from the power law at large bending curvature but can still be primely fitted by the power law at small to moderate curvature (Fig. 2c). DFT-based molecular dynamic simulations at $300 \mathrm{~K}$ show that bending-induced extension is only slightly smaller at room temperature than at $0 \mathrm{~K}$ (supplemental materials, Fig. S7 (footnote 2)). It is also found that the bending rigidity or modulus may be overestimated without considering the accordion effect. For example, the obtained bending moduli of monolayered BP from our DFT results are 1.55 and $7.09 \mathrm{eV}$ along the armchair and zigzag directions, respectively (Fig. 2d), but the values become 4.07 and $8.92 \mathrm{eV}$ when the bending-induced extension is constrained to zero (supplemental materials, Fig. S8 (footnote 2)).

\section{Conclusion}

In conclusion, we revealed a novel intrinsic mechanical behavior of $2 \mathrm{D}$ crystals - bending-induced extension, or the accordion effect. Our comprehensive DFT-based firstprinciples calculations showed that it is evocable owing to the specific atomic thinness monolayer bonding structures and the localization of the density of electrons on the compressive side of the monolayer constrains the compressive deformations resulting in an overall tensile deformation perpendicular to the bending moment. This accordion effect is intrinsic to all of the investigated 2D crystals. The accordion effect can be best described by the simple power law with an exponent of around 2, making 2D crystals more promising for novel applications, especially for mechanoelectrooptical devices.
Acknowledgements This project was supported by the 973 program (Grants 2012CB937500, 2013CB932604), the National Natural Science Foundation of China (Grants 51535005, 51472117, 11021262, 11172303, 11132011), and the Fundamental Research Funds for the Central Universities (Grant NP2013309). We thank X.F. Liu for helpful discussions and W.H. Tang for help in drawing some of the geometrical figures.

\section{References}

1. Novoselov, K.S., Fal, V.I., Colombo, L., et al.: A roadmap for graphene. Nature 490, 192-200 (2012)

2. Ferrari, A.C., Bonaccorso, F., Fal'Ko, V., et al.: Science and technology roadmap for graphene, related two-dimensional crystals, and hybrid systems. Nanoscale 7, 4598-4810 (2015)

3. Kim, K.S., Zhao, Y., Jang, H., et al.: Large-scale pattern growth of graphene films for stretchable transparent electrodes. Nature $\mathbf{4 5 7}$, 706-710 (2009)

4. Georgiou, T., Jalil, R., Belle, B.D., et al.: Vertical field-effect transistor based on graphene-WS2 heterostructures for flexible and transparent electronics. Nat. Nanotech. 8, 100-103 (2013)

5. Jones, A.M., Yu, H., Ross, J.S., et al.: Spin-layer locking effects in optical orientation of exciton spin in bilayer $\mathrm{WSe}_{2}$. Nat. Phys. 10, 130-134 (2014)

6. Li, L., Yu, Y., Ye, G.J., et al.: Black phosphorus field-effect transistors. Nat. Nanotech. 9, 372-377 (2014)

7. Pereira, V.M., Neto, A.C., Liang, H.Y., et al.: Geometry, mechanics, and electronics of singular structures and wrinkles in graphene. Phys. Rev. Lett. 105, 156603 (2010)

8. Klimov, N.N., Jung, S., Zhu, S., et al.: Electromechanical properties of graphene drumheads. Science 336, 1557-1561 (2012)

9. Bakr, W.S., Peng, A., Tai, M.E., et al.: Probing the superfluidto-mott insulator transition at the single-atom level. Science 329, 544-547 (2010)

10. Lee, C., Wei, X., Kysar, J.W., et al.: Measurement of the elastic properties and intrinsic strength of monolayer graphene. Science 321, 385-388 (2008)

11. Bertolazzi, S., Brivio, J., Kis, A.: Stretching and breaking of ultrathin $\mathrm{MoS}_{2}$. ACS Nano 5, 9703-9709 (2011)

12. Ma, R., Bando, Y., Sasaki, T.: Directly rolling nanosheets into nanotubes. J. Phys. Chem. B 108, 2115-2119 (2004)

13. Liu, X., Pan, D., Hong, Y., et al.: Bending poisson effect in twodimensional crystals. Phys. Rev. Lett. 112, 205502 (2014)

14. Zhao, X., Liu, Y., Inoue, S., et al.: Smallest carbon nanotube is 3 $\AA$ in diameter. Phys. Rev. Lett. 92, 125502 (2004)

15. Anderoglu, O., Misra, A., Wang, H., et al.: Epitaxial nanotwinned $\mathrm{Cu}$ films with high strength and high conductivity. Appl. Phys. Lett. 93, 083108 (2008)

16. Pan, D., Wang, T.-C., Guo, W.: Bending-induced phase transition in monolayer black phosphorus. Chin. Phys. B 24, 086401 (2015) 
17. Zólyomi, V.: Theoretical investigation of small diameter carbon nanotubes. [Ph.D. Thesis], Eötvös University, Budapest (2005)

18. Shi, X., Peng, B., Pugno, N.M., et al.: Stretch-induced softening of bending rigidity in graphene. Appl. Phys. Lett 100, 191913 (2012)

19. Cadelano, E., Giordano, S., Colombo, L.: Interplay between bending and stretching in carbon nanoribbons. Phys. Rev. B 81, 144105 (2010)

20. Koskinen, P.: Electromechanics of twisted graphene nanoribbons. Appl. Phys. Lett. 99, 013105 (2011)

21. Jiang, L., Guo, W.L.: Analytical solutions for elastic binary nanotubes of arbitray chirality. Acta. Mech. Sin. 32, 1045-1056 (2016)

22. Yeh, N.-C., Hsu, C.-C., Teague, M.L., et al.: Nanoscale strain engineering of graphene and graphene-based devices. Acta. Mech. Sin. 32, 497-509 (2016)

23. Hou, J., Yin, Z., Zhang, Y., et al.: Structure dependent elastic properties of supergraphene. Acta. Mech. Sin. 32, 684-689 (2016)

24. Kohn, W., Sham, L.J.: Self-consistent equations including exchange and correlation effects. Phys. Rev. 140, A1133-A1138 (1965)
25. Kresse, G., Furthmüller, J.: Efficient iterative schemes for ab initio total-energy calculations using a plane-wave basis set. Phys. Rev. B 54, 11169 (1996)

26. Kresse, G., Furthmüller, J.: Efficiency of ab-initio total energy calculations for metals and semiconductors using a plane-wave basis set. Comput. Mater. Sci. 6, 15-50 (1996)

27. Perdew, J.P., Burke, K., Ernzerhof, M.: Generalized gradient approximation made simple. Phys. Rev. Lett. 77, 3865 (1996)

28. Blöchl, P.E.: Projector augmented-wave method. Phys. Rev. B 50, 17953-17979 (1994)

29. Kresse, G., Hafner, J.: Ab initio molecular dynamics for liquid metals. Phys. Rev. B 47, 558 (1993)

30. Kresse, G., Joubert, D.: From ultrasoft pseudopotentials to the projector augmented-wave method. Phys. Rev. B 59, 1758 (1999)

31. Reddy, J.N.: Theory and Analysis of Elastic Plates and Shells. CRC Press, Boca Raton (2006) 